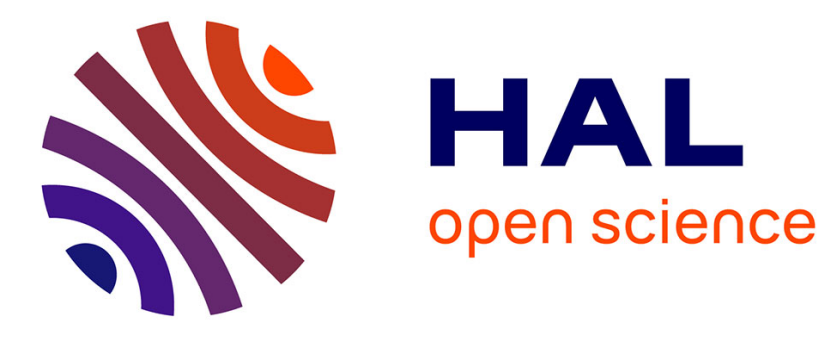

\title{
Electrical Impedance Tomography by Elastic Deformation
}

Habib Ammari, Eric Bonnetier, Yves Capdeboscq, Mickael Tanter, Mathias Fink

\section{- To cite this version:}

Habib Ammari, Eric Bonnetier, Yves Capdeboscq, Mickael Tanter, Mathias Fink. Electrical Impedance Tomography by Elastic Deformation. SIAM Journal on Applied Mathematics, 2008, 68 (6), pp.1557-1573. 10.1137/070686408 . hal-00128366v2

\section{HAL Id: hal-00128366 https://hal.science/hal-00128366v2}

Submitted on 4 Feb 2007

HAL is a multi-disciplinary open access archive for the deposit and dissemination of scientific research documents, whether they are published or not. The documents may come from teaching and research institutions in France or abroad, or from public or private research centers.
L'archive ouverte pluridisciplinaire HAL, est destinée au dépôt et à la diffusion de documents scientifiques de niveau recherche, publiés ou non, émanant des établissements d'enseignement et de recherche français ou étrangers, des laboratoires publics ou privés. 


\title{
ELECTRICAL IMPEDANCE TOMOGRAPHY BY ELASTIC DEFORMATION
}

\author{
H. AMMARI , E. BONNETIER , Y. CAPDEBOSCQ , M. TANTER , AND M. FINK
}

\begin{abstract}
This paper presents a new algorithm for conductivity imaging. Our idea is to extract more information about the conductivity distribution from data that have been enriched by coupling impedance electrical measurements to localized elastic perturbations. Using asymptotics of the fields in the presence of small volume inclusions, we relate the pointwise values of the energy density to the measured data, through a nonlinear PDE. Our algorithm is based on this PDE and takes full advantage of the enriched data. We give numerical examples that illustrate the performance and the accuracy of our approach
\end{abstract}

\section{INTRODUCTION}

Electrical impedance tomography (EIT) technique has been an active research topic since the early 1980s. In EIT, one measures the boundary voltages due to multiple injection currents to reconstruct images of the conductivity distribution. However, these boundary voltages are insensitive to a local change of the conductivity distribution and the relation between them is highly nonlinear.

Medical imaging has been one of the important application areas of EIT. Indeed, biological tissues have different electrical properties that change with cell concentration, cellular structure, and molecular composition. Such changes of electrical properties are the manifestations of structural, functional, metabolic, and pathological conditions of tissues, and thus provide valuable diagnostic information.

For practitioners, the practicality of EIT is of great interest: It is a low cost and portable technology, which can be used for real time monitoring. However, it suffers from poor spatial resolution and accuracy, a well-known feature of inverse problems. This motivated us to look for a new way of incorporating more information in EIT data, without altering the cost and portability of the data acquisition, which would yet improve the resolution of the reconstructed images.

The classical image reconstruction algorithms view EIT as an optimization problem. An initial conductivity distribution is iteratively updated, so as to minimize the difference between measured and computed boundary voltages. This kind of method was first introduced in EIT by Yorkey, Webster, and Tompkins [33]. Numerous variations and improvements followed, which include utilization of a priori information, and various forms of regularization [32]. This approach is quite greedy in computational time, yet produces images with deceivingly poor accuracy and spatial resolution.

In the 1980's, Barber and Brown [11] introduced a back-projection algorithm, that was the first fast and efficient algorithm for EIT, although it provides images 
with very low resolution. Since this algorithm is inspired from computed tomography, it can be viewed as a generalized Radon transform method [30].

A third technique is dynamical electrical impedance imaging, developed by the Rensselaer impedance tomography group [19], to produce images of changes in conductivity due to cardiac or respiratory functions. Its main idea consists in viewing the conductivity as the sum of a static term (the background conductivity of the human body) plus a perturbation (the change of conductivity caused by respiration or by heart beats). The mathematical problem here is to visualize the perturbation term by an EIT system. Although this algorithm provides accurate images if the initial guess of the background conductivity is good, its resolution does not completely satisfy practitioners especially when screening for breast cancer.

Recently, a commercial system called TransScan TS2000 (TransScan Medical, Ltd, Migdal Ha'Emek, Israel) has been released for adjunctive clinical uses with Xray mammography in the diagnostic of breast cancer. Interestingly, the TransScan system is similar to the frontal plane impedance camera that initiated EIT research early in 1978. The mathematical model of the TransScan can be viewed as a realistic or practical version of the general EIT system, so any theory developed for this model can be applied to other areas in EIT, especially to detection of anomalies. In the TransScan, a patient holds a metallic cylindrical reference electrode, through which a constant voltage of 1 to $2.5 \mathrm{~V}$, with frequencies spanning $100 \mathrm{~Hz}-100 \mathrm{KHz}$, is applied. A scanning probe with a planar array of electrodes, kept at ground potential, is placed on the breast. The voltage difference between the hand and the probe induces a current flow through the breast, from which information about the impedance distribution in the breast can be extracted.

Using a simplified dipole method, Assenheimer et Scholz gave a physical interpretation of the white spots in TransScan images.

More recently, taking advantage of the smallness of the anomalies to be detected, Ammari et al. [8] analyzed trans-admittance data, for the detection of breast cancer using the TransScan system. Their model assumes that breast tissues can be considered homogeneous, at least near the surface, where the planar array of electrodes is attached, and that the lesion to be detected is located near the surface. In [8], the authors developed better ways of interpreting TransScan images, which improve accuracy. They also derived a multi-frequency approach to handle the case where the background conductivity is inhomogeneous and not known a priori.

This latter work relies on asymptotic expansions of the fields when the medium contains inclusions of small volume, a technique that has proven useful in many other contexts. Such asymptotics have been investigated in the case of the conduction equation [18, 17, 13, 4, 15], the operator of elasticity [3, 7], the Helmholtz equation or the Maxwell system $[34,9,6]$. See the book [5] and its list of references. The remarkable feature of this technique, is that it allows a stable and accurate reconstruction of the location and of the geometric features of the inclusions, even for moderately noisy data.

Since all the present EIT technologies are only practically applicable in feature extraction of anomalies, improving EIT calls for innovative measurement techniques that incorporate structural information. A very promising direction of research is the recent magnetic resonance imaging technique, called current density imaging, which measures the internal current density distribution. See the breakthrough 
work by Seo and his group [27, 28, 24]. However, this technique has a number of disadvantages, among which the lack of portability and a potentially long imaging time. Moreover, it uses an expensive magnetic resonance imaging scanner.

The aim of this paper is to propose another mathematical direction for future EIT research in view of biomedical applications, without eliminating the most important merits of EIT (real time imaging, low cost, portability). Our method is based on the simultaneous measurement of an electric current and of acoustic vibrations induced by ultrasound waves. Its intrinsic resolution depends on the size of the focal spot of the acoustic perturbation, and thus our method should provide high resolution images.

Let us now formulate our problem. We first recall that, in mathematical terms, EIT consists in recovering the conductivity map of a $2 \mathrm{D}$ or $3 \mathrm{D}$ body $\Omega$, from measuring the voltage response to one or several currents applied on the boundary. In practice, a set of electrodes is attached to the body. One or several currents $\phi_{i}, 1 \leq i \leq I$, are applied to one or several electrodes, and the corresponding voltage potentials $f_{i}, 1 \leq i \leq I$, are recorded on the others. Denoting by $\gamma(x)$ the unknown conductivity, the voltage potential $u_{i}$ solve the conduction problem

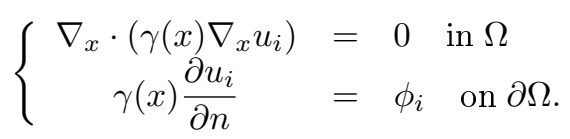

The problem of impedance tomography is the inverse problem of recovering the coefficients $\gamma$ of the elliptic conduction PDE, knowing one or more currentto-voltage pairs $\left(\phi_{i}, f_{i}:=\left.u_{i}\right|_{\partial \Omega}\right)$.

The core idea of our approach is to extract more information about the conductivity from data that has been enriched by coupling the electric measurements to localized elastic perturbations. More precisely, we propose to perturb the medium during the electric measurements, by focusing ultrasonic waves on regions of small diameter inside the body. Using a simple model for the mechanical effects of the ultrasound waves, we show that the difference between the measurements in the unperturbed and perturbed configurations is asymptotically equal to the pointwise value of the energy density at the center of the perturbed zone. In practice, the ultrasounds impact a spherical or ellipsoidal zone, of a few millimeters in diameter. The perturbation should thus be sensitive to conductivity variations at the millimeter scale, which is the precision required for breast cancer diagnostic.

By scanning the interior of the body with ultrasound waves, given an applied current $\phi_{i}$, we obtain data from which we can compute $\mathcal{S}_{i}(x):=\gamma(x)\left|\nabla u_{i}(x)\right|^{2}$, in an interior sub-region of $\Omega$. The new inverse problem is now to reconstruct $\gamma$ knowing $\mathcal{S}_{i}$ for $i=1, \ldots, I$.

The goal of this work is threefold: Firstly, we show that taking measurements while perturbing the medium with ultrasound waves is asymptotically equivalent to measuring $\mathcal{S}_{i}$. To this end, we consider the zone $\omega$ deformed by the ultrasound wave as a small volume perturbation of the background potential $\gamma$. We then relate the difference between the perturbed and unperturbed potentials on the boundary to the conductivity at the center of $\omega$, asymptotically as $|\omega| \rightarrow 0$. This is our main idea: the ultrasound waves create localized perturbations that allow us, using the method of asymptotic expansions of small volume inclusions, to probe within the medium. 


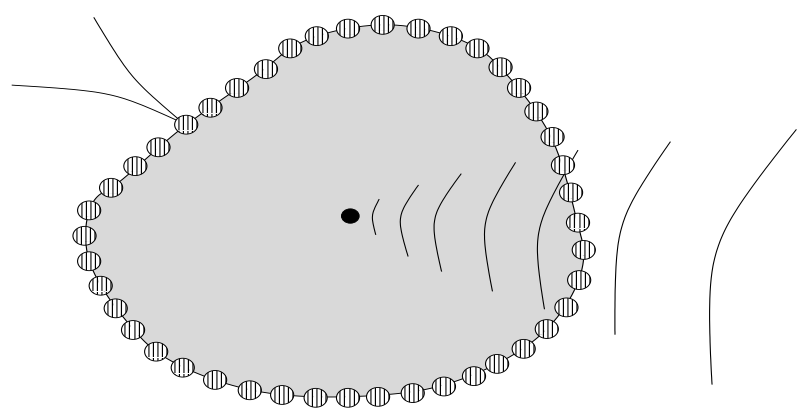

FiguRE 2.1. The experimental setup.

Secondly, noting that the potential $u_{i}$ satisfies the following nonlinear PDE (the 0-Laplacian)

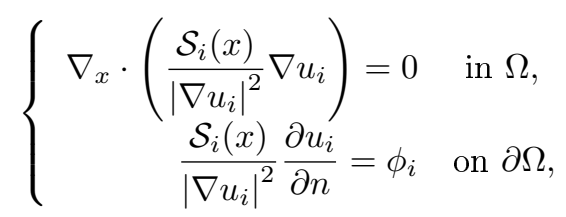

we propose a numerical method to compute solutions $\tilde{u}_{i}$ to $(1.2)$ and then an approximate conductivity $\tilde{\gamma}=\mathcal{S}_{i} /\left|\nabla \tilde{u}_{i}\right|^{2}$, using two currents (i.e., $I=2$ ). Recall that an appropriate choice of $\phi_{i}$ insures that $\nabla u_{i} \neq 0$ for all $x \in \Omega$. See $[1,2,31,21]$.

Thirdly, our algorithm, as the one originally developed in [25] for current density imaging, requires data measured with two boundary currents for which the flux densities (the gradient of the voltage potentials) are locally orthogonal (or try to be). We show numerically that this algorithm is able to capture details of the conductivity map up to the precision of the underlying finite element mesh, and thus proves very effective.

The paper is organized as follows. In the next section, we describe the physical model, and the collection of experimental data on the boundary. Section 3 explains how, given a current $\phi_{i}$, the values of $\mathcal{S}_{i}(x)$ can be approximated using this data. In section 4 , we describe the numerical method for reconstruction of $\gamma$ using two applied currents. Numerical examples that illustrate the performance and the accuracy of this method are presented in that section. The paper ends with a short discussion.

\section{IMPEDANCE TOMOGRAPHY PERTURBED BY ULTRASOUND WAVES}

2.1. Description of the experiment. The goal of the experiment is to obtain an impedance map inside a solid with millimetric precision.

An objet (a domain $\Omega$ ) is electrically probed: One or several currents are imposed on the surface and the induced potentials are measured on the boundary (see fig 2.1). At the same time, a spherical region of a few millimeters in the interior of $\Omega$ is mechanically excited by ultrasonic waves, which dilate this region.

The measurements are made as the focus of the ultrasounds scans the entire domain. Several sets of measurements can be obtained by varying the ultrasound waves amplitudes and the applied currents. 
2.2. Physical modelling of the effect of ultrasonic waves on the conductivity. Within each (small) spherical volume, the conductivity is assumed to be constant per volume unit. At a point $x \in \Omega$, within a ball $B$ of volume $V_{B}$, the electric conductivity $\gamma$ is defined in terms of a density $\rho$ as

$$
\gamma(x)=\rho(x) V_{B} .
$$

The ultrasonic waves induce a small elastic deformation of the sphere $B$. If this deformation is isotropic, the material points of $B$ occupy a volume $V_{B}^{p}$ in the perturbed configuration, which at first order is equal to

$$
V_{B}^{p}=V_{B}\left(1+3 \frac{\Delta r}{r}\right),
$$

where $r$ is the radius of the ball $B$ and $\Delta r$ is the variation of the radius due to the elastic perturbation. As $\Delta r$ is proportional to the amplitude of the ultrasonic wave, we obtain a proportional change of the deformation. Using two different ultrasonic waves with different amplitudes but with the same spot, it is therefore easy to compute the ratio $V_{B}^{p} / V_{B}$. As a consequence, the perturbed electrical conductivity $\gamma^{p}$ satisfies

$$
\forall x \in \Omega, \quad \gamma^{p}(x)=\rho(x) V_{B}^{p}=\gamma(x) \nu(x),
$$

where $\nu(x)=V_{B}^{p} / V_{B}$ is a known function. We make the following realistic assumptions:

(H1): the ultrasonic wave expands the zone it impacts, and changes its conductivity:

$$
\forall x \in \Omega, \nu(x)>1 .
$$

(H2): The perturbation is not too small:

$$
\nu(x)-1 \gg V_{B}
$$

2.3. Mathematical modelling of the effect of ultrasonic waves on the conductivity. We denote by $u$ the voltage potential induced by a current $\phi$, in the absence of ultrasonic perturbations. It is given by

$$
\left\{\begin{array}{c}
\nabla_{x} \cdot\left(\gamma(x) \nabla_{x} u\right)=0 \text { in } \Omega, \\
\gamma(x) \frac{\partial u}{\partial n}=\phi \text { on } \partial \Omega
\end{array}\right.
$$

with the convention that $\int_{\partial \Omega} u=0$. We assume that the conductivity $\gamma$ is bounded above and below by positive constants

$$
0<c<\gamma(x)<C<+\infty \text { a.e. } x \in \Omega .
$$

Further, we suppose that the conductivity $\gamma$ is known close to the boundary of the domain, so that ultrasonic probing is limited to interior points $x$ such that

$$
\operatorname{dist}(x, \partial \Omega) \geq d_{0},
$$

where $d_{0}$ is very large compared to the radius of the spot of the ultrasonic perturbation. We denote the corresponding open set $\Omega_{1}$. We denote by $u_{\omega}(x), x \in \Omega$, the voltage potential induced by a current $\phi$, in the presence of ultrasonic perturbations localized in a domain $\omega$ of volume $|\omega|$. The voltage potential $u_{\omega}$ is a solution to

$$
\left\{\begin{array}{c}
\nabla_{x} \cdot\left(\gamma_{\omega}(x) \nabla_{x} u_{\omega}(x)\right)=0 \text { in } \Omega, \\
\gamma(x) \frac{\partial u_{\omega}}{\partial n}=\phi \text { on } \partial \Omega,
\end{array}\right.
$$


with the notation

$$
\gamma_{\omega}(x)=\gamma(x)\left[1+1_{\omega}(x)(\nu(x)-1)\right]
$$

where $1_{\omega}$ is the characteristic function of the domain $\omega$. In the next section, we show how comparing $u_{\omega}$ and $u$ on $\partial \Omega$ provides information about the conductivity.

\section{Asymptotic ReCovery of the CONDUCtivity}

As the zone deformed by the ultrasound wave is small, we can view it as a small volume perturbation of the background conductivity $\gamma$, and seek an asymptotic expansion of the boundary values of $u_{\omega}-u$.

For each $i=1, \ldots, I$, let $\zeta_{\omega}^{i}$ be the solution to

$$
\left\{\begin{array}{l}
\nabla_{x} \cdot\left(\gamma_{\omega}(x) \nabla_{x} \zeta_{\omega}^{i}\right)=\nabla_{x} \cdot\left(\gamma(x) \nabla_{x} x_{i}\right) \text { in } \Omega \\
\gamma(x) \frac{\partial \zeta_{\omega}^{i}}{\partial n}=\gamma(x) \frac{\partial x_{i}}{\partial n} \text { on } \partial \Omega, \text { with } \int_{\Omega} \zeta_{\omega}^{i}=0
\end{array}\right.
$$

Corresponding to $\zeta_{\omega}^{i}$, we define $\zeta^{i}=x_{i}-c_{i}$ where $c_{i}$ is a constant, in the unperturbed case.

The following proposition is a variant of a compactness result proved in [15] which we recall for completeness. In contrast to previous work, the proof we give here only requires boundedness of the conductivity $\gamma_{\omega}$.

Proposition 3.1. Consider a sequence of sets $\omega \subset \subset \Omega$, such that $\frac{1}{|\omega|} \mathbf{1}_{\omega}$ converges in the sense of measures to a probability measure $d \mu$ as $|\omega|$ tends to zero. Then, the correctors $\frac{1}{|\omega|} \mathbf{1}_{\omega} \frac{\partial \zeta_{\omega}^{i}}{\partial x_{j}}$ converge in the sense of measures to $M_{i j} d \mu$, where $M_{i j}$ is a matrix-valued function.

Furthermore, it satisfies

$$
\left\|\nabla\left(\zeta_{\omega}^{i}-\zeta^{i}\right)\right\|_{L^{2}(\Omega)^{d}} \leq C|\omega|^{1 / 2} \text {, and }\left\|\zeta_{\omega}^{i}-\zeta^{i}\right\|_{L^{2}(\Omega)} \leq C|\omega|^{\frac{1}{2}+\kappa},
$$

where the constants $\kappa>0$ and $C>0$ depend only on $\Omega_{1}, \sup _{\Omega}\left|\gamma_{\omega}\right|$, and inf $\operatorname{in}_{\Omega}\left|\gamma_{\omega}\right|$.

Proof. The bounds on $\nabla\left(\zeta_{\omega}^{i}-\zeta^{i}\right)$ and $\left(\zeta_{\omega}^{i}-\zeta^{i}\right)$ are a direct consequence of Lemma A.1, if we remark that, inside the domain $\Omega, \zeta_{\omega}^{i}-\zeta^{i}$ satisfies

$$
\nabla_{x} \cdot\left(\gamma_{\omega}(x) \nabla_{x}\left(\zeta_{\omega}^{i}-\zeta^{i}\right)\right)=-\nabla_{x} \cdot\left(\mathbf{1}_{\omega}\left(\gamma_{p}-\gamma\right) \nabla_{x} x_{i}\right) \text { in } \Omega .
$$

As for the existence of a limit (and its additional properties) we refer to [15].

One of the key elements of our method is the following representation formula.

Proposition 3.2. Assume that $u \in W^{2, \infty}(\omega)$. Then,

$$
\int_{\partial \Omega}\left(u_{\omega}-u\right) \phi d \sigma=|\omega| \int_{\Omega}\left(\gamma_{\omega}(x)-\gamma(x)\right) M_{\omega} \nabla u \nabla u d x+O\left(|\omega|^{1+\kappa}\right) .
$$

The exponent $\kappa$ only depends on $\Omega_{1}, \sup _{\Omega}\left|\gamma_{\omega}\right|$ and $\inf _{\Omega}\left|\gamma_{\omega}\right|$. The remainder term has the form

$$
\left|O\left(|\omega|^{1+\kappa}\right)\right| \leq C|\omega|^{1+\kappa}\|\nabla u\|_{L^{\infty}(\omega)^{d}}\left\|\nabla^{2} u\right\|_{L^{\infty}(\omega)^{d \times d}}
$$

where $C$ depends only on $\Omega_{1}, \sup _{\Omega}\left|\gamma_{\omega}\right|$, and $\inf _{\Omega}\left|\gamma_{\omega}\right|$. Finally, the matrix valued function $M_{\omega}$ is given by

$$
\left(M_{\omega}\right)_{i j}(x)=\frac{1}{|\omega|} \mathbf{1}_{\omega}(x) \frac{\partial}{\partial x_{j}} \zeta_{\omega}^{i}(x) \quad \text { a.e. } x \in \Omega_{1} .
$$


This is, globally, not a new result. This representation formula, and the proof presented here has been already obtained by Capdeboscq and Vogelius, it is Theorem 1 in [15]. The only difference with Theorem 1 of [15] is that the regularity required on $u$ is investigated more in depth. Note that gobally, $u$ satisfies the minimal requirement $u \in H^{1}(\Omega)$. Additional regularity on $u$ is only required within $\omega$ (in particular, the quality of the representation formula is not affected). We also note that if $\omega$ is a disk in the two-dimensional case then (see for instance [17])

$$
M_{\omega}=\frac{1}{|\omega|} \mathbf{1}_{\omega}(x) \frac{\nu-1}{\nu+1} I_{2}
$$

where $I_{2}$ is the unit matrix. The following corollary holds.

Corollary 3.3. Assume that the dimension $d=2$, that the perturbed area $\omega$ is a disk centered at $z$, and that $u \in W^{2, \infty}(\omega)$. Then, we have

$$
\begin{aligned}
\int_{\partial \Omega}\left(u_{\omega}-u\right) \phi d \sigma & =\int_{\omega} \gamma(x) \frac{(\nu(x)-1)^{2}}{\nu(x)+1} \nabla u \cdot \nabla u d x+O\left(|\omega|^{1+\kappa}\right) \\
& =|\nabla u(z)|^{2} \int_{\omega} \gamma(x) \frac{(\nu(x)-1)^{2}}{\nu(x)+1} d x+O\left(|\omega|^{1+\kappa}\right) .
\end{aligned}
$$

Therefore, if $\gamma$ is $\mathcal{C}^{0, \alpha}(\omega)$, with $0 \leq \alpha \leq \kappa$, we have

$$
\gamma(z)|\nabla u(z)|^{2}=\mathcal{S}(z)+O\left(|\omega|^{\alpha}\right) \quad(\text { or o }(1) \text { if } \alpha=0)
$$

where the function $\mathcal{S}(z)$ is defined by

$$
\mathcal{S}(z)=\left(\int_{\omega} \frac{(\nu(x)-1)^{2}}{\nu(x)+1} d x\right)^{-1} \int_{\partial \Omega}\left(u_{\omega}-u\right) \phi d \sigma .
$$

We emphasize that $\mathcal{S}(z)$ represents a known function, as the second term on the right-hand side of (3.2) is exactly the measured data. We also note that the formula (3.1) is only valid where $|\mathcal{S}(z)| \gg|\omega|^{\kappa}$.

Proof of Proposition 3.2. This proof follows the Proof of Lemma 2 in [15]. From (2.2) and (2.3), we obtain using Green's formula that, for all $w \in H^{1}(\Omega)$,

$$
\int_{\partial \Omega} u_{\omega} \phi d \sigma=\int_{\Omega} \gamma \nabla u_{\omega} \nabla u d x, \quad \text { and } \quad \int_{\partial \Omega} u \phi d \sigma=\int_{\Omega} \gamma_{\omega} \nabla u_{\omega} \nabla u d x
$$

Consequently,

$$
\begin{aligned}
\int_{\partial \Omega}\left(u_{\omega}-u\right) \phi d \sigma & =\int_{\Omega}\left(\gamma-\gamma_{\omega}\right) \nabla u_{\omega} \nabla u d x \\
& =\int_{\omega}\left(\gamma-\gamma_{\omega}\right) \nabla u_{\omega} \nabla u d x
\end{aligned}
$$

Notice that $u_{\omega}-u$ satisfies a homogenous Neumann boundary condition, and verifies

$$
\nabla \cdot\left(\gamma_{\omega} \nabla\left(u_{\omega}-u\right)\right)=-\nabla \cdot\left(1_{\omega}\left(\gamma_{\omega}-\gamma\right) \nabla u\right) \text { in } \Omega .
$$

Since $u \in W^{1, \infty}(\omega)$, we can invoke again Lemma A.1 to obtain that

$\left\|\nabla\left(u_{\omega}-u\right)\right\|_{L^{2}(\Omega)^{d}} \leq C|\omega|^{1 / 2}\|\nabla u\|_{L^{\infty}(\omega)^{d}}$, and $\left\|u_{\omega}-u\right\|_{L^{2}(\Omega)} \leq C|\omega|^{\frac{1}{2}+\kappa}\|\nabla u\|_{L^{\infty}(\omega)^{d}}$, 
where the constants $C, \kappa$ depend only on $\Omega_{1}, \sup _{\Omega}\left|\gamma_{\omega}\right|$ and $\inf _{\Omega}\left|\gamma_{\omega}\right|$. For all $\phi \in$ $W^{1, \infty}(\Omega)$, we now compute

$$
\begin{aligned}
\int_{\Omega} \gamma_{\omega} \nabla\left(u_{\omega}-u\right) \cdot \nabla \zeta_{\omega}^{i} \phi d x & =\int_{\Omega} \gamma_{\omega} \nabla\left(\left(u_{\omega}-u\right) \phi\right) \cdot \nabla \zeta_{\omega}^{i} d x \\
& -\int_{\Omega} \gamma_{\omega}\left(u_{\omega}-u\right) \nabla \phi \cdot \nabla \zeta_{\omega}^{i} d x \\
& =\int_{\Omega} \gamma \nabla\left(\left(u_{\omega}-u\right) \phi\right) \nabla \zeta^{i} d x+r_{1} \\
& =\int_{\Omega} \gamma \nabla\left(u_{\omega}-u\right) \nabla \zeta^{i} \phi d x+r_{2} .
\end{aligned}
$$

The remainder term is given by

$$
\begin{aligned}
r_{2} & =\int_{\Omega} \gamma\left(u_{\omega}-u\right) \nabla \phi \nabla \zeta^{i} d x-\int_{\Omega} \gamma_{\omega}\left(u_{\omega}-u\right) \nabla \phi \nabla \zeta_{\omega}^{i} d x \\
& =O\left(|\omega|^{1+\kappa}\right) .
\end{aligned}
$$

Here, by $O\left(|\omega|^{1+\kappa}\right)$ we denote a quantity that is bounded by $C\|\nabla u\|_{L^{\infty}(\omega)^{d}}\|\nabla \phi\|_{L^{\infty}(\Omega)^{d}}|\omega|^{1+\kappa}$, where $C$ depends only on $\Omega_{1}, \sup _{\Omega}\left|\gamma_{\omega}\right|$, and $\inf _{\Omega}\left|\gamma_{\omega}\right|$.

On one hand, we have

$$
\begin{aligned}
\int_{\Omega} \gamma_{\omega} \nabla\left(u_{\omega}-u\right) \cdot \nabla \zeta_{\omega}^{i} \phi d x & =\int_{\Omega} \gamma_{\omega} \nabla\left(u_{\omega}-u\right) \cdot \nabla\left(\zeta_{\omega}^{i} \phi\right) d x \\
& -\int_{\Omega} \gamma_{\omega} \nabla\left(u_{\omega}-u\right) \cdot \nabla \phi \zeta_{\omega}^{i} d x, \\
& =\int_{\omega}\left(\gamma-\gamma_{\omega}\right) \nabla u \cdot \nabla\left(\zeta_{\omega}^{i} \phi\right) d x \\
& -\int_{\Omega} \gamma_{\omega} \nabla\left(u_{\omega}-u\right) \cdot \nabla \phi \zeta^{i} d x+O\left(|\omega|^{1+\kappa}\right), \\
& =\int_{\omega}\left(\gamma-\gamma_{\omega}\right) \nabla u \cdot \nabla \zeta_{\omega}^{i} \phi d x \\
& +\int_{\Omega}\left(\gamma \nabla u-\gamma_{\omega} \nabla u_{\omega}\right) \cdot \nabla \phi \zeta^{i} d x+O\left(|\omega|^{1+\kappa}\right) .
\end{aligned}
$$

On the other hand, we have

$$
\begin{aligned}
\int_{\Omega} \gamma \nabla\left(u_{\omega}-u\right) \cdot \nabla \zeta^{i} \phi d x & =\int_{\Omega} \gamma \nabla\left(u_{\omega}-u\right) \cdot \nabla\left(\zeta^{i} \phi\right) d x \\
& -\int_{\Omega} \gamma \nabla\left(u_{\omega}-u\right) \cdot \nabla \phi \zeta^{i} d x, \\
& =\int_{\omega}\left(\gamma-\gamma_{\omega}\right) \nabla u_{\omega} \cdot \nabla \zeta^{i} \phi d x \\
& +\int_{\Omega}\left(\gamma \nabla u-\gamma_{\omega} \nabla u_{\omega}\right) \cdot \nabla \phi \zeta^{i} d x .
\end{aligned}
$$

We have obtained that, for all $i=1, \ldots, d$,

(3.4) $\int_{\omega}\left(\gamma-\gamma_{p}\right) \frac{\partial u_{\omega}}{\partial x_{i}} \phi d x=\sum_{j=1}^{d} \int_{\omega}\left(\gamma-\gamma_{\omega}\right) \frac{\partial u}{\partial x_{j}} \cdot \frac{\partial}{\partial x_{j}} \zeta_{\omega}^{i} \phi d x+O\left(|\omega|^{1+\kappa}\right)$,

with

$$
\left|O\left(|\omega|^{1+\kappa}\right)\right| \leq C|\omega|^{1+\kappa}\|\nabla u\|_{L^{\infty}(\omega)^{d}}\|\nabla \phi\|_{L^{\infty}(\Omega)^{d}}
$$


where $C$ is a constant that depends only on $\Omega_{1}$, $\sup _{\Omega}\left|\gamma_{\omega}\right|$, and $\inf _{\Omega}\left|\gamma_{\omega}\right|$. Choose $\phi_{i}=\frac{\partial}{\partial x_{i}} u * \eta_{\epsilon}$ in $\omega_{\epsilon}=\{x \in \omega$ s.t. $\operatorname{dist}(x, \partial \omega)>\epsilon\}$ where $\eta$ is the standard mollifier, and let $\Phi_{\epsilon}$ be defined as

$$
\Phi_{\epsilon}=\left(\frac{\partial}{\partial x_{1}} u * \eta_{\epsilon}, \ldots, \frac{\partial}{\partial x_{d}} u * \eta_{\epsilon}\right)
$$

Summing (3.4) over $i$ we obtain

$$
\int_{\omega}\left(\gamma-\gamma_{p}\right) \nabla u_{\omega} \cdot \nabla \Phi_{\epsilon} d x=|\omega| \int_{\omega}\left(\gamma-\gamma_{p}\right) \nabla u \cdot\left(M_{\omega} \Phi_{\epsilon}\right) d x+O\left(|\omega|^{1+\kappa}\right),
$$

where

$$
O\left(|\omega|^{1+\kappa}\right) \leq C\|\nabla u\|_{L^{\infty}(\omega)^{d}}\left\|\nabla \Phi_{\epsilon}\right\|_{L^{\infty}(\omega)^{d}} \leq C\|\nabla u\|_{L^{\infty}(\omega)^{d}}\left\|\nabla^{2} u\right\|_{L^{\infty}(\omega)^{d}} .
$$

By passing to the limit in $\epsilon$ and using (3.3), the proof of the proposition is complete.

\section{Reconstruction using the 0-Laplacian Formulation}

In view of deriving an approximation for the conductivity $\gamma$ inside $\Omega_{1}$, we introduce the following equation

$$
\left\{\begin{aligned}
\nabla \cdot\left(\frac{\mathcal{S}(x)}{|\nabla u|^{2}} \nabla u\right) & =0 & & \text { in } \Omega, \\
\frac{\mathcal{S}(x)}{|\nabla u|^{2}} \frac{\partial u}{\partial n} & =\phi & & \text { on } \partial \Omega .
\end{aligned}\right.
$$

We emphasize that $\mathcal{S}$ is a known function, constructed from the measured data (3.2). Consequently, all the parameters entering in equation (4.1) are known.

Our approach uses measurements $\mathcal{S}_{1}$ and $\mathcal{S}_{2}$ obtained using two distinct currents, $\phi_{1}$ and $\phi_{2}$. We choose this pair of current patterns to have $\nabla u_{1} \times \nabla u_{2} \neq 0$ for all $x \in \Omega$, where $u_{i}, i=1,2$, is the solution to (1.1). See [31,21] for an evidence of the possibility of such a choice.

We start from an initial guess for the conductivity $\gamma$, and solve the corresponding Dirichlet conductivity problem

$$
\left\{\begin{array}{rc}
\nabla \cdot\left(\gamma \nabla u_{0}\right)=0 & \text { in } \Omega \\
u_{0}=\psi & \text { on } \partial \Omega
\end{array}\right.
$$

The data $\psi$ is the Dirichlet data measured as a response to the current $\phi$ (say $\left.\phi=\phi_{1}\right)$ in absence of elastic deformation.

The discrepancy between the data and our guessed solution is

$$
\epsilon_{0}:=\frac{\mathcal{S}(x)}{\left|\nabla u_{0}\right|^{2}}-\gamma .
$$

We then introduce a corrector, $u_{c}$, computed as the solution to

$$
\left\{\begin{array}{rc}
\nabla \cdot\left(\gamma \nabla u_{c}\right)=-\nabla \cdot\left(\varepsilon_{0} \nabla u_{0}\right) & \text { in } \Omega \\
u_{c}=0 & \text { on } \partial \Omega,
\end{array}\right.
$$

and update the conductivity

$$
\gamma:=\frac{\mathcal{S}(x)-2 \gamma \nabla u_{c} \cdot \nabla u_{0}}{\left|\nabla u_{0}\right|^{2}} .
$$

We iteratively update the conductivity, alternating directions (i.e., with $\phi=\phi_{2}$ ). 
To study the efficiency of this approach, we have tested this method on various problems and domains, using the partial differential equation solver FreeFem $++[22]$. We present here one such test. The domain $\Omega$ is a disk of radius 8 centered at the origin, which contains three inclusions, an ellipse, an L-shaped domain and a triangle, so as to image a convex object, a non-convex object, and an object with a smooth boundary.

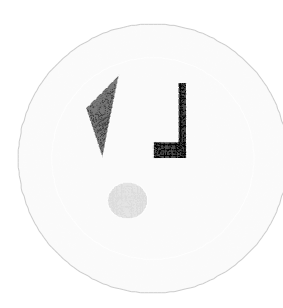

Figure 4.1. Conductivity distribution.

The background conductivity is equal to 0.5 , the conductivity takes the values 2 in the triangle, 0.75 in the ellipse and 2.55 in the $\mathrm{L}$-shaped domain. We purposedly chose values corresponding to small and large contrast with the background. Note that our approach is perturbative, thus the smaller the contrast the easier the detection: The choice of a significant contrast was not made to highlight the objects, but rather to make the reconstruction more challenging.

Figure 4.2 shows the result of the reconstruction when perfect measures (with 'infinite' precision) are available. We use two different boundary potentials, $\psi=$ $x /|x|$ and $\psi=y /|y|$. The initial guess is depicted on the left: it is equal to 1 inside the disk of radius 6 centered at the origin, and equal to the supposedly known conductivity $\gamma=0.5$ near the boundary (outside the disk of radius 6 ). The two central pictures represent the collected data, $\mathcal{S}(x)$ for $\psi=x /|x|$ on the left and $\mathcal{S}(x)$ for $\psi=y /|y|$ on the right. Given the values of the contrast, we remark that although one can 'see' the triangle and the L-shape inclusions on these plots, the circle is hardly noticeable. On the far right, the reconstructed conductivity is represented: it perfectly matches the target.
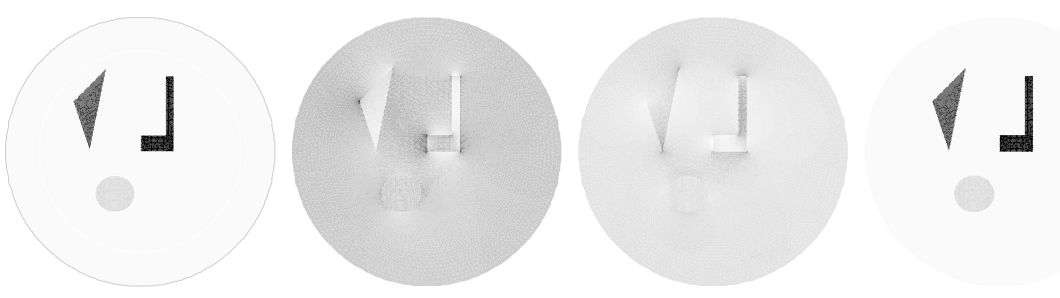

Figure 4.2. Reconstruction test. From left to right, the initial guess, the collected data $\mathcal{S}(x /|x|$ and $y /|y|)$ and the reconstructed conductivity. 
In Figure 4.3, the error is represented as a function of the number of iterations. The dotted curve is a plot of the (intrinsic) error estimator $\max _{x \in \Omega} \epsilon_{n}(x)$, given by (4.2). The curve with diamond symbols depicts the $L^{1}$-norm $a_{1}(n)$ of the true error between the reconstructed conductivity $\tilde{\gamma}$ and the original one:

$$
a_{1}(n):=\int_{\Omega}|\tilde{\gamma}-\gamma| d x .
$$

Note that the abcissa is represented in logarithmic scale, thus the convergence seems exponential.

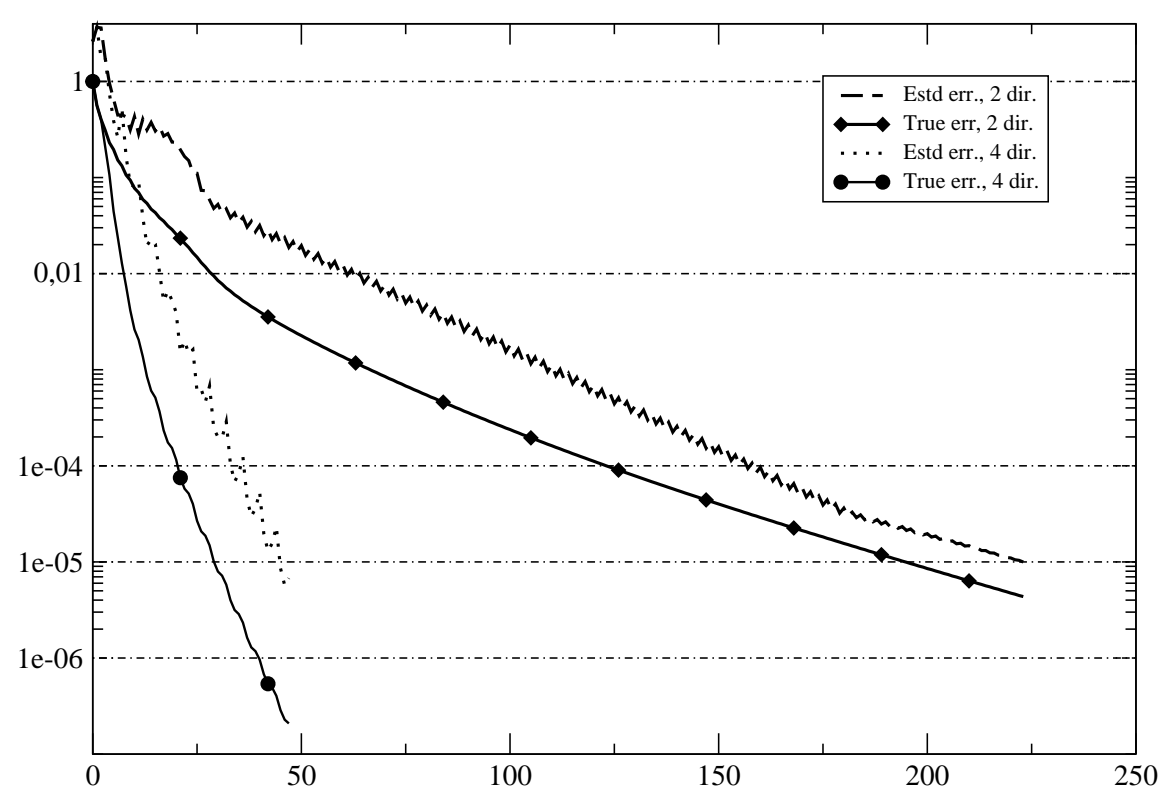

FiguRE 4.3. Convergence results. The curve labeled "Estd err., 2 dir." (resp. "True err., 2 dir.") corresponds to the estimated error (resp. true error) when two directions are used. The curves "Estd err., 4 dir." and "True err., 4 dir." are the errors computed when 4 directions are used.

The other two curves correspond to the same computations, but four directions are used instead of two: $x /|x|, y /|y|,(x+y) /|x+y|,(x-y) /|x-y|$. Note that this does not require more measurements because of the linear dependence on the boundary condition: it is merely a change in the algorithm. The same level of error for $\epsilon_{n}$ is reached in 45 iterations, instead of 222. The same experiment, with a contrast 5 time smaller, converges in less than ten iterations.

We also considered imperfect data. In Figure 4.4 we follow the same procedure but now assume that the data was measured at the nodes of a regular mesh on the disk, with 50,100,200 and 400 boundary points. To give an idea of the scale of the mesh compared to the objects, the projections of the conductivity that we wish to recover are represented in the left column. The two central columns depict the collected data. The column on the far right shows the obtained reconstructions. 


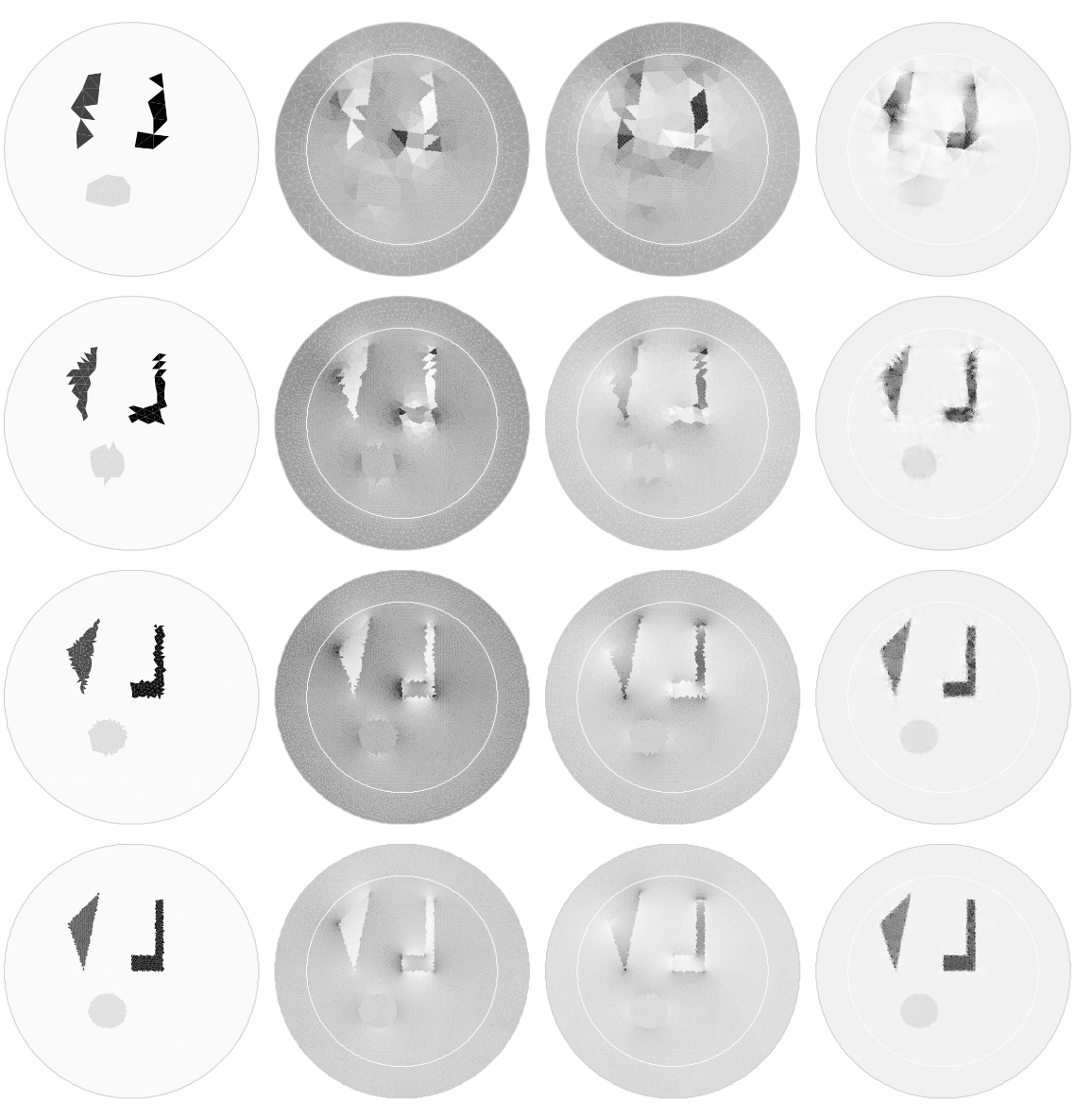

FiguRE 4.4. Reconstruction tests. From top to bottom, using a regular mesh with 50,100,200, and 400 boundary points. From left to right, the initial guess, the collected data $\mathcal{S}$ (for $x /|x|$ and $y /|y|$ ) and the reconstructed conductivity.

To accelerate the computations, we used the four direction variant of the algorithm. The error as a function of the iterations is represented in Figure 4.5. The curves with symbols represent the $L^{1}$ norm of the true error, whereas the dashed line is the $L^{2}$ norm of the estimated error $\epsilon$, for the most precise mesh. Although the estimated error does not decrease noticeably, the true error does. The reconstructed image is obtained after ten iterations and does not change noticeably henceforth. This calls for further refinements of the algorithm, such as adapted stepsizes and adapted meshes. Improvements are indeed possible, and will be the subject of a future publication. As it stands, the algorithm already provides reconstructions comparable in accuracy to those of projected conductivity. Naturally, the sharp corners are easily localized, but the smooth elliptic shape is also accurately reconstructed, even at the coarsest scale. 


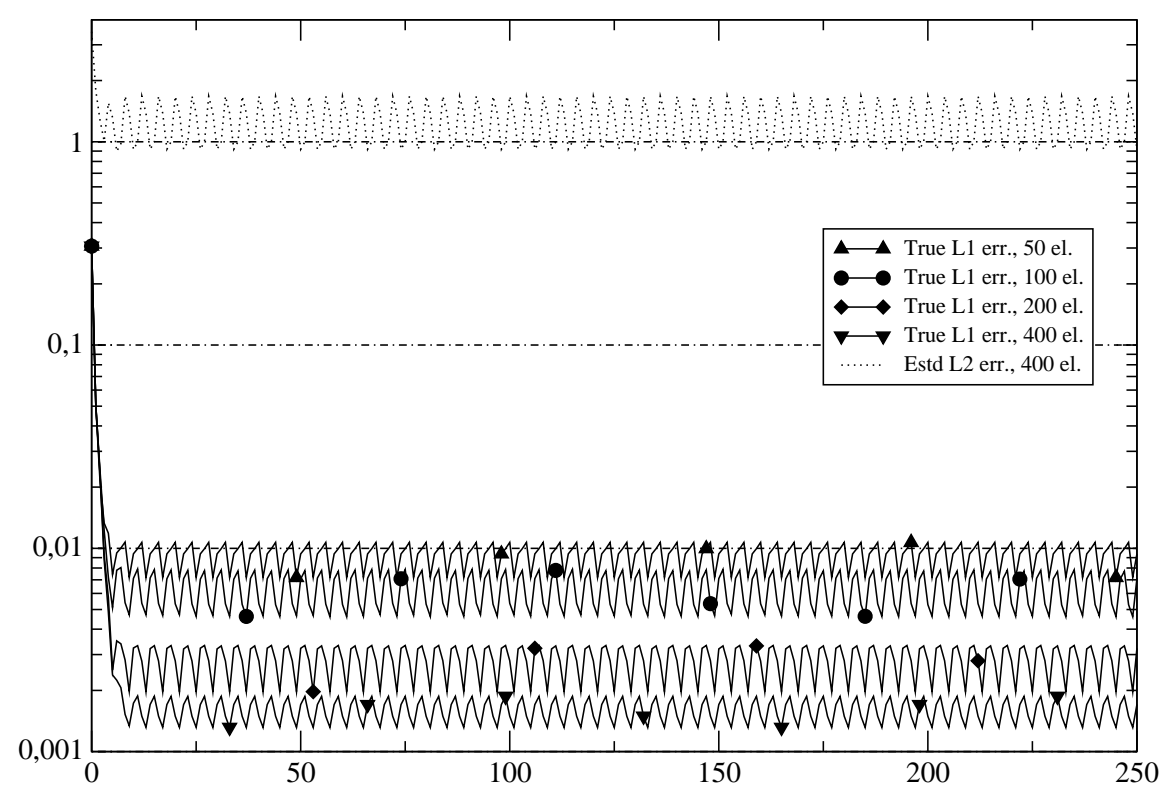

FiguRE 4.5. Convergence results. The curves labeled "True L1 err." correspond to the $L^{1}$ norm of the discrepancy between the real and reconstructed conductivity. The curve labeled "Estd L2 err., 400 el." represents the estimated error for the regular mesh designed with 400 boundary points.

\section{Concluding Remarks}

We have proposed a new technique for conductivity imaging, which consists in perturbing the medium during the electric measurements, by focusing ultrasonic waves on regions of small diameter inside the body. We derived an approximation of the conductivity using small volume asymptotics and obtained a non linear PDE for the potential, in terms of the measured data. Based on this PDE, we proposed a new algorithm for the reconstruction of the conductivity distribution, which proves remarkably accurate.

Motivated by the practical limitations of EIT, we intend to pursue the present investigation in the following directions:

(i) Study the reconstruction capabilities of this method when only partial data, measured on a small portion $\Gamma$ of the boundary, is available.

(ii) Study the dependence of the algorithm on the global geometry of $\Omega$.

(iii) Study the sensitivity of the method to limitations on the intensities of the applied voltages, as electrical safety regulations limit the amount of the total current that patients can sustain.

Moreover, we also intend to address some of the mathematical questions raised by this imaging approach, among which the uniqueness for solutions to the PDE (1.2), the uniqueness of the inverse problem of recovering the conductivity distribution with two measurements, and the convergence analysis of the reconstruction algorithm. 


\section{ApPENDix A}

Lemma A.1. Let $a \in L^{\infty}(\Omega)$ be a positive function, satisfying $C_{0}>a>c_{0}>0$, and let $F \in L^{\infty}(\Omega)^{d}$. Let $V$ be a closed subset of $H^{1}(\Omega)$ such that

$$
H_{0}^{1}(\Omega) \subset V \subset H^{1}(\Omega)
$$

Assume that $\phi \in V$ is such that

$$
\nabla \cdot(a \nabla \phi)=\nabla \cdot\left(1_{\omega}(x) F\right) \text { in } \Omega .
$$

Suppose that $\Omega$ contains a subset of $\Omega^{\prime} \subset \Omega$ of class $\mathcal{C}^{2}$, such that $\operatorname{dist}\left(\Omega^{\prime}, \partial \Omega\right)>$ $d_{0}>0$, and such that $\omega \subset \Omega^{\prime}$, or alternatively that $\Omega$ is a cube and that $\phi$ is periodic on that cube. Then,

$$
\|\nabla \phi\|_{L^{2}(\Omega)^{d}} \leq \frac{1}{\sqrt{c_{0}}}|\omega|^{1 / 2}\|F\|_{L^{\infty}(\Omega)^{d}} .
$$

Furthermore, there exists $\kappa>0$ and $C>0$, two positive constants depending only on $\Omega^{\prime}, d_{0}, c_{0}$, and $C_{0}$, such that

$$
\|\phi\|_{L^{2}(\Omega)} \leq C|\omega|^{\frac{1}{2}+\kappa}\|F\|_{L^{\infty}(\Omega)^{d}} .
$$

At this point, let us emphasize the fact that the background is not required to be smooth. The proof uses Meyers' Theorem [29].

Proof. We shall now prove Lemma A.1 for $V=H^{1}(\Omega)$ or $V=H_{0}^{1}(\Omega)$. Integrating (A.1) against $\phi$, we obtain

$$
\int_{\Omega} a \nabla \phi \cdot \nabla \phi d x=\int_{\Omega} 1_{\omega} F \cdot \nabla \phi d x .
$$

Thus, using Cauchy-Schwarz inequality, we get (A.2). Define $\psi \in H^{1}(\Omega)$ as the unique solution to

$$
\begin{aligned}
-\nabla \cdot(a \nabla \psi) & =\phi \text { in } \Omega \\
\psi & =0 \text { on } \partial \Omega .
\end{aligned}
$$

Choose $f \in \mathcal{C}_{0}^{\infty}(\Omega)$ to be a cut-off function such that $f \equiv 1$ on $\Omega^{\prime}$, and $0 \leq f \leq 1$. According to Meyers' Theorem [29] (see also [12, p. 35-45]), there exists a $\eta>0$ depending only on $\Omega, c_{0}, C_{0}$, and $f$ such that $\psi \in W^{1,2+\eta}\left(\Omega^{\prime}\right)$, and we have

$$
\|\nabla(\psi f)\|_{L^{2+\eta}(\Omega)} \leq C\|\phi\|_{L^{2+\eta}(\Omega)} .
$$

Using Gagliardo-Nirenberg inequality,

$$
\begin{aligned}
\|\phi\|_{L^{2+\eta}(\Omega)} & \leq C\|\phi\|_{L^{2}(\Omega)}^{\alpha}\|\nabla \phi\|_{L^{2}(\Omega)^{d}}^{1-\alpha} \text { with } \alpha=\frac{\eta}{2+\eta} \\
& \leq C\|\phi\|_{L^{2}(\Omega)}^{\alpha}|\omega|^{\frac{1}{2+\eta}} .
\end{aligned}
$$

We then compute

$$
\begin{aligned}
\int_{\Omega} \phi^{2} d x & =\int a \nabla \psi \nabla \phi \\
& =\int 1_{\omega} F \nabla \psi \\
& \leq\|F\|_{L^{\infty}(\Omega)}|\omega|^{\frac{1+\eta}{2+\eta}}\|\nabla(f \psi)\|_{L^{2+\eta}(\Omega)} \\
& \leq C\|F\|_{L^{\infty}(\Omega)}|\omega|\|\phi\|_{L^{2}(\Omega)}^{\alpha} .
\end{aligned}
$$


Consequently,

$$
\|\phi\|_{L^{2}(\Omega)} \leq C\|F\|_{L^{\infty}(\Omega)}|\omega|^{\frac{1}{2}+\frac{\eta}{2(4+\eta)}}
$$

and therefore, choosing $\kappa=\frac{\eta}{2(4+\eta)}$ concludes the proof.

\section{REFERENCES}

[1] G. Alessandrini, V. Isakov, and J. Powell, Local uniqueness in the inverse conductivity problem with one measurement, Trans. Amer. Math. Soc., 347 (1995), 3031-3041.

[2] G. Alessandrini and R. Magnanini, The index of isolated critical points and solutions of elliptic equations in the plane, Ann. Scuola Norm. Sup. Pisa Cl. Sci. (4), 19 (1992), 567-589.

[3] C. Alves and H. Ammari, Boundary integral formulae for the reconstruction of imperfections of small diameter in an elastic medium. SIAM J. Appl. Math. (2001), 62, 94-106.

[4] H. Ammari and H. Kang, High-order terms in the asymptotic expansions of the steady-state voltage potentials in the presence of inhomogeneities of small diameter. SIAM J. Math. Anal., 34 (2003), 1152-1166.

[5] H. Ammari and H. Kang, Reconstruction of Small Inhomogeneities from Boundary Measurements, Lecture Notes in Mathematics, Volume 1846, Springer-Verlag, Berlin, 2004.

[6] H. Ammari and H. Kang, Boundary layer techniques for solving the Helmholtz equation in the presence of small inhomogeneities, J. Math. Anal. Appl., 296 (2004), 190-208.

[7] H. Ammari, H. Kang, G. Nakamura, and K. Tanuma, Complete asymptotic expansions of solutions of the system of elastostatics in the presence of an inclusion of small diameter and detection of an inclusion, J. Elasticity, 67 (2002), 97-129.

[8] H. Ammari, O. Kwon, J.K. Seo, and E.J. Woo, Anomaly detection in T-scan trans-admittance imaging system, SIAM J. Appl. Math., 65 (2004), 252-266.

[9] H. Ammari, M. Vogelius and D. Volkov, Asymptotic formulas for perturbations in the electromagnetic fields due to the presence of inhomogeneities of small diameter II. The full Maxwell equations. J. Math. Pures Appl., 80 (2001), 769-814.

[10] M. Assenheimer, O. Laver-Moskovitz, D. Malonek, D. Manor, U. Nahliel, R. Nitzan, and A. Saad, The T-scan technology: Electrical impedance as a diagnostic tool for breast cancer detection, Physiol. Meas., 22 (2001), 1-8.

[11] D.C. Barber and B.H. Brown, Applied potential tomography, J. Phys. Sci. Instrum., 17 (1984), 723-733.

[12] A. Bensoussan, J.L. Lions, and G. Papanicolaou, Asymptotic Analysis for Periodic Structures, Studies in Mathematics and its Applications, 5. North-Holland Publishing Co., AmsterdamNew York, 1978.

[13] E. Beretta, E. Francini and M. Vogelius, Asymptotic formulas for steady state voltage potentials in the presence of thin inhomogeneities. A rigorous error analysis, J. Math. Pures Appl., 82 (2003), 1277-1301.

[14] M. Bruhl, M. Hanke, and M.S. Vogelius, A direct impedance tomography algorithm for locating small inhomogeneities, Numer. Math., 93 (2003), 635-654.

[15] Y. Capdeboscq and M. Vogelius, A general representation formula for boundary voltage perturbations caused by internal conductivity inhomogeneities of low volume fraction. Math. Modelling Num. Anal., 37 (2003), 159-173.

[16] Y. Capdeboscq and M. Vogelius, Optimal asymptotic estimates for the volume of internal inhomogeneities in terms of multiple boundary measurements, Math. Modelling Num. Anal., 37 (2003), 227-240.

[17] D.J. Cedio-Fengya, S. Moskow and M. Vogelius, Identification of conductivity imperfections of small diameter by boundary measurements. Continuous dependence and computational reconstruction, Inverse Problems, 14 (1998), 553-595.

[18] A. Friedman and M.S. Vogelius, Identification of small inhomogeneities of extreme conductivity by boundary measurements: a theorem on continuous dependence, Arch. Rat. Mech. Anal., 105 (1989), 299-326.

[19] M. Cheney, D. Isaacson, and J.C. Newell, Electrical impedance tomography, SIAM Rev., 41 (1999), 85-101.

[20] V.A. Cherepenin, A. Karpov, A. Korjenevsky, V. Kornienko, A. Mazaletskaya, D. Mazourov, and D. Meister, A 3D electrical impedance tomography (EIT) system for breast cancer detection, Physiol. Meas., 22 (2001), 9-18. 
[21] E. Fabes, H. Kang, and J.K. Seo, Inverse conductivity problem with one measurement: Error estimates and approximate identification for perturbed disks, SIAM J. Math. Anal., 30 (1999), 699-720.

[22] F. Hecht, O. Pironneau, K. Ohtsuka, A. Le Hyaric, FreeFem++, http://www.freefem.org/ (2007).

[23] D. Holder, Clinical and Physiological Applications of Electrical Impedance Tomography, UCL Press, London, 1993.

[24] Y. J. Kim, O. Kwon, J.K. Seo, and E.J. Woo, Uniqueness and convergence of conductivity image reconstruction in magnetic resonance electrical impedance tomography, Inverse Problems, 19 (2003), 1213-1225.

[25] S. Kim, O. Kwon, J.K. Seo, and J.R. Yoon, On a nonlinear partial differential equation arising in magnetic resonance electrical impedance imaging, SIAM J. Math. Anal., 34 (2002), $511-526$.

[26] R.V. Kohn and M.S. Vogelius, Determining conductivity by boundary measurements, Comm. Pure Appl. Math., 37 (1984), 289-298.

[27] O. Kwon, J.K. Seo, and J.R. Yoon, A real-time algorithm for the location search of discontinuous conductivities with one measurement, Comm. Pure Appl. Math., 55 (2002), 1-29.

[28] O. Kwon, E.J. Woo, J.R. Yoon, and J.K. Seo, Magnetic resonance electrical impedance tomography (MREIT): simulation study of J -substitution algorithm IEEE Trans. Biomed. Eng., 49 (2002), 160-167.

[29] N.G. Meyers, An $L^{p}$-estimate for the gradient of solutions of second order elliptic divergence equations, Ann. Scuola Norm. Sup. Pisa, 3 (1963), 189-206.

[30] F. Santosa and M.S. Vogelius, A backprojection algorithm for electrical impedance imaging, SIAM J. Appl. Math., 50 (1990), 216-243.

[31] J.K. Seo, A uniqueness result on inverse conductivity problem with two measurements, J. Fourier Anal. Appl., 2 (1996), 227-235.

[32] E.J. Woo, J.G. Webster, and W.J. Tompkins, A robust image reconstruction algorithm and its parallel implementation in electrical impedance tomography, IEEE Trans. Med. Imag., 12 (1993), 137-146.

[33] T. Yorkey, J. Webster, and W. Tompkins, Comparing reconstruction algorithms for electrical impedance tomography, IEEE Trans. Biomed. Engr., 34 (1987), 843-852.

[34] M. Vogelius and D. Volkov, Asymptotic formulas for perturbations in the electromagnetic fields due to the presence of inhomogeneities, Math. Model. Numer. Anal., 34 (2000), 723748 .

LABORATOIRE ONDES ET ACOUSTIQUE, CNRS, ESPCI\& PARIS VII, 10 RUE VAUQUELIN, 75231 PARIS CEDEX 05, FRANCE

E-mail address: HABIB. AMMARI@POLYTECHN IQUE.FR

UNIVERSITÉ JOSEPH FOURIER, LABORATOIRE DE MODÉLISATION ET CALCUL, BP 53, 38041, GRENOBLE CEDEX 9, FRANCE

E-mail address: ERIC.BONNETIER@IMAG.FR

LMV, UNIVERSITÉ DE VERSAILLES-SAINT QUENTIN, CNRS, VERSAILLES, FRANCE

E-mail address: YVES.CAPDEBOSCQ@UVSQ.FR

LABORATOIRE ONDES ET ACOUSTIQUE, CNRS, ESPCI \& PARIS VII, 10 RUE VAUQUELIN, 75231 PARIS CEDEX 05, FRANCE

E-mail address: MIKAEL.TANTER@ESPCI.FR

LABORATOIRE ONDES ET ACOUSTIQUE, CNRS, ESPCI \& PARIS VII, 10 RUE VAUQUELIN, 75231 PARIS CEDEX 05, FRANCE

E-mail address: MATHIAS.FINK@ESPCI.FR 